\title{
Curious Case of a Foldable Intraocular Lens that did not Unfold
}

\section{ABSTRACT}

Phacoemulsification is a modern technique of cataract surgery that allows the removal of cataractous lens and implantation of a foldable Intraocular Lens (IOL) through a small sub $3 \mathrm{~mm}$ incision. For a good visual outcome, it is imperative that the IOL is well centered and its haptics unfold properly inside the capsular bag and keep it distended. However, at times the IOL haptics may not unfold properly leading to adverse visual symptoms. Herein, Authors present an unusual case of a 61-year-old elderly male with no other associated ocular or systemic illness, who presented nine months after cataract surgery in his left eye, with both haptics of the IOL unusually folded and the edge of one haptic encroaching into the pupillary area leading to visual symptoms. As multiple attempts to dial the haptics into the capsular bag were unsuccessful, likely due to strong capsular adhesions, the unfolded haptics were placed in the sulcus with attempted optic capture. The postoperative period was uneventful with BCVA of 20/20 at one year. The current case highlights the need to be vigilant about the proper positioning of the haptics at the end of routine cataract surgery and the need for early intervention in the event of any complication.

Keywords: Haptic, Phacoemulsification, Redialing

\section{CASE REPORT}

A 61-year-old male presented to the outpatient department with complains of not having satisfactory vision and seeing an annoying shadow on one side from his left eye. He had undergone cataract surgery with IOL implantation in his left eye nine months back elsewhere and had been observing these symptoms since around one month after surgery. Medical records of the surgery or details about the type of IOL implanted were not available; however, on enquiry from the concerned hospital where he had undergone the surgery, it was reported to be a hydrophilic IOL routinely supplied under the government scheme. His Uncorrected Visual Acuity (UCVA) was 20/60 and Best Corrected Visual Acuity (BCVA) was 20/20 with a manifest refraction of $-1.00 \mathrm{D}$ (Dioptres)-3.00 $\mathrm{D} \times 100$ degree. Undilated slit lamp examination was unremarkable [Table/Fig-1a], but on narrow slit beam illumination an unusual appearance of IOL was seen on the nasal side [Table/Fig-1b]. The anterior chamber was quiet and formed. Examination after dilation of the pupil showed both haptics folded in an unusual manner, with an intact capsulorhexis margin and an intact posterior capsule [Table/Fig2a,b]. The capsulorhexis size ranged from 5.5 to $6 \mathrm{~mm}$ in different meridians. There were a few folds in the posterior capsule and focal areas of dense fibrosis were apparent adjacent to the optic haptic junctions. Fundus examination was unremarkable. Examination of the other eye and systemic examination were also unremarkable.
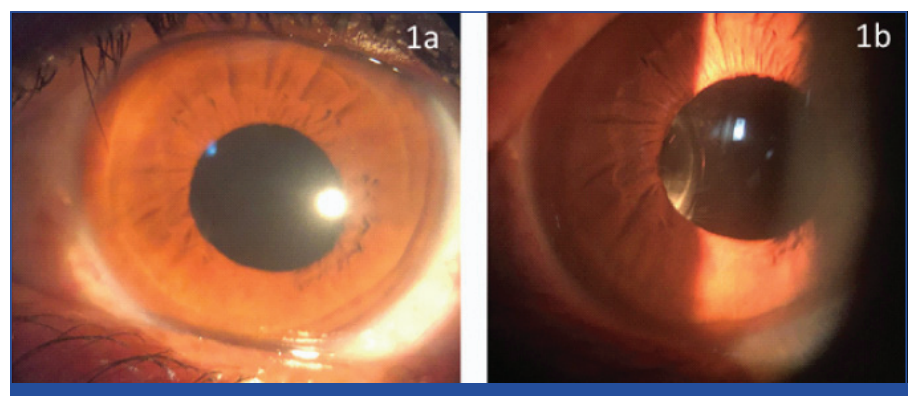

[Table/Fig-1]: Undilated slit lamp biomicroscopy in diffuse illumination show normal appearance of IOL in the pupillary area (a); and narrow slit beam examination reveal an unusual appearance of IOL on the nasal side (b).

Surgery for unfolding and redialing of the IOL haptics into the desired position inside the capsular bag was planned. Under peribulbar anaesthesia, two side port incisions were made at 1 O'clock and
5 O'clock positions at the limbus. Viscoelastic was injected into the anterior chamber and an attempt was made to separate the anterior and posterior capsules all around with gentle dissection using a blunt spatula. Subsequently, the trapped and folded haptics were gently freed from the capsular adhesions with the help of two Sinskey hooks and the IOL was brought anteriorly into the sulcus. Multiple attempts to dial the IOL haptics into the capsular bag were unsuccessful on account of strong focal adhesions between the anterior and posterior capsules near the equatorial zone. Once it was apparent that it would not be possible to dial the IOL into the capsular bag, dialing procedure was abandoned to avoid stress on the zonules or to risk a posterior capsular rupture with vigorous IOL manipulations. The haptics were eventually placed in the sulcus with attempted optic capture into the capsulorhexis margin. The bimanual irrigation and aspiration system was used to remove the viscoelastic, the side ports were hydrated and subconjunctival injection of dexamethasone and gentamicin was given. Postoperative treatment consisted of topical Moxifloxacin $(0.5 \%)$ eye drops four times daily, Prednisolone acetate eye drops $(1 \%)$ six times daily and Nepafenac $(0.1 \%)$ eye drops three times daily for two weeks. Prednisolone eye drops were gradually tapered over the next four weeks. The postoperative period was uneventful with one week postoperative UCVA of 20/40 and BCVA of 20/20 with a manifest refraction of $-1.00 \mathrm{D}-1.00 \mathrm{D} \times 24$ degrees. He continued to maintain this BCVA at one year follow-up with resolution of ocular symptoms and without any evidence of intraocular inflammation. Slit lamp examination showed a well centered IOL with open haptics and an intact posterior capsule [Table/Fig-3a,b].

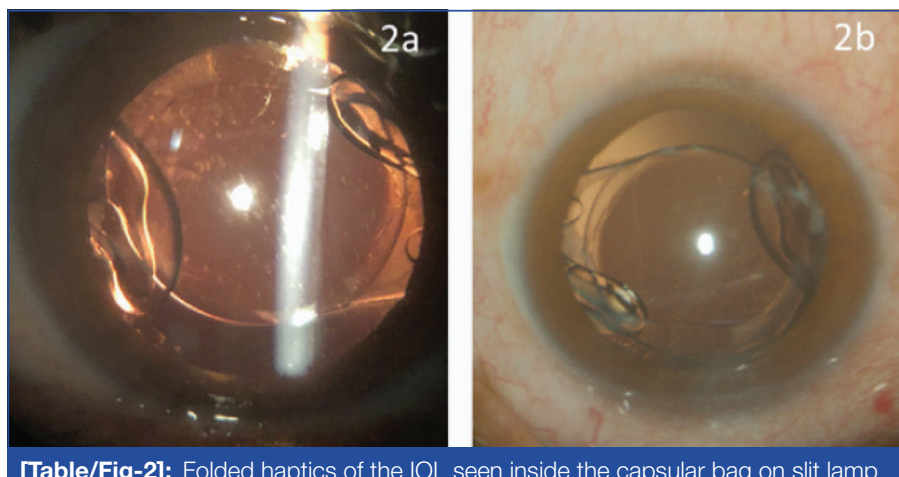

[Table/Fig-2]: Folded haptics of the IOL seen inside the capsular bag on slit lamp examination (a); and as seen intraoperatively through the surgical microscope (b). 

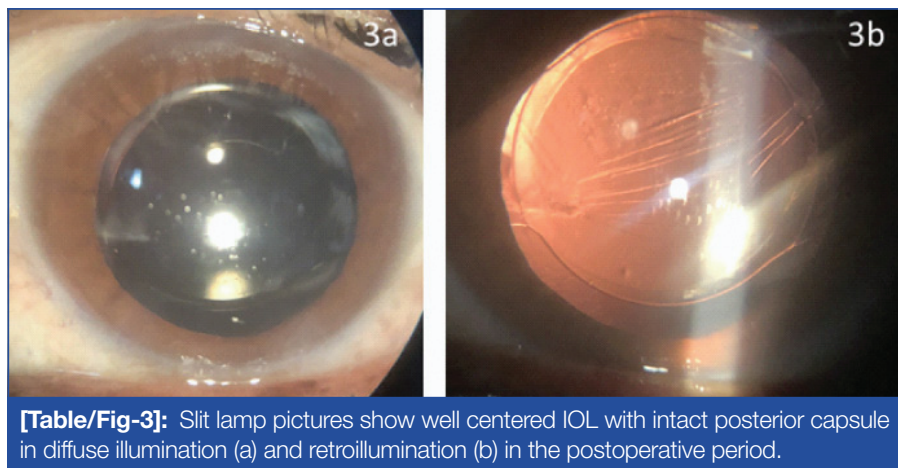

in diffuse illumination (a) and retroillumination (b) in the postoperative period.

\section{DISCUSSION}

The current case highlights an unusual complication of a singlepiece foldable $\mathrm{OOL}$ and the difficulties faced in the attempted surgical procedure to dial its haptics into the desired anatomical location. Although reports of poor refractive outcome after an uneventful cataract surgery due to tilted IOL or unfolded haptics have been published in literature [1-3]; these cases had involvement of only one haptic, and we could not find any report with both haptics of IOL stuck and folded like in present case, in literature review.

The exact reason for such an unusual appearance of IOL was not clear, however, we speculate that it could be a case where the IOL might have been inadvertently left in this folded position during the surgery itself wherein intraoperative miosis obscured the view of haptics and surgeon wrongly assumed that IOL haptics had been dialed into position. Dada T (2004) reported a case of small pupil phacoemulsification in a diabetic patient where the leading haptic was found to be folded under the optic leading to IOL tilt on the first postoperative day which had to be surgically corrected [1]. Dada T et al. (2005), reported another case of a 58-year-old male who presented with poor visual recovery three weeks after phacoemulsification surgery. The authors found IOL tilt on slit lamp examination which was confirmed to be due to folded haptic on ultrasound biomicroscopy. The patient underwent surgery to release the folded haptic into the capsular bag with resolution of symptoms. The authors advocated use of two Sinskey hooks to retract the iris and check haptics configuration intraoperatively in small pupils to avoid this complication [2]. Merchant K and Stirling R reported a case of a 76-year-old male who presented with gradual visual disturbance three months after standard phacoemulsification surgery. On examination, the inferior haptic was found to be folded on itself with associated capsular contraction. The patient was operated again and the folded haptic was trimmed. There was good outcome at two months postoperative examination without any destabilisation of $\mathrm{IOL}$ which was attributed to its firm adherence to the contracted capsule around it [3]. Although this patient was non-diabetic and had been operated elsewhere, intraoperative miosis leading to obstructed view of the haptics cannot be ruled out as the likely cause. Also, in the current case both the haptics were folded which made the idea of trimming of haptics untenable.

Other causes of such an appearance of $\mathrm{IOL}$ could be due to capsular fibrosis associated with conditions like pseudo exfoliation syndrome, diabetes, uveitis, myotonic dystrophy or retinitis pigmentosa [4], however, the current patient did not have any findings known to be associated with these disorders. Also, the capsulorhexis size at presentation in the current case was large enough to rule out any associated capsular phimosis or exuberant fibrosis.
The other surgical option in the current case would have been an IOL exchange with implantation of a3-piece IOLin the sulcus. Implantation of a single piece IOL in the sulcus is usually not recommended as it has been reported to cause multiple complications including uveitis, glaucoma, hyphema and vitreous haemorrhage, however, these studies usually include complicated surgeries with associated rupture of posterior capsule and use of a hydrophobic square edge IOL in the sulcus [5-7]. In contrast, there are other studies in literature that report that single piece $\mathrm{OOL}$ have been implanted safely in the sulcus without any untoward adverse effects $[8,9]$. In the current case, there was no evidence of any intraocular inflammation over a follow-up of one year. This could be attributed to preservation of the integrity of posterior capsule during the surgery as well as to the hydrophilic nature of the IOL material which can be presumed to be more iris friendly as compared to square edged haptics of a hydrophobic IOL. Also, IOL exchange may not be a routine and easy procedure for many ophthalmologists and can potentially lead to complications of zonular dialysis, posterior capsular tears, damage to corneal endothelium and difficult prediction of postoperative refraction [10]. Furthermore, it can always be done as an elective procedure later if the need arises.

\section{CONCLUSION(S)}

The current case highlights the need to be vigilant about the proper positioning of the haptics at the end of routine cataract surgery. With intraoperative miosis, the IOL may appear to be in position in the centre of the pupil, as was likely the case in this patient and in such situations, retraction of iris in various directions may be helpful in confirming the proper positioning of the IOL and its haptics. The current case also highlights the difficulty in dialing the haptics into the capsular bag in the late postoperative period due to strong adhesions between the two capsules thus emphasising on the need for early intervention in such cases.

\section{REFERENCES}

[1] Dada T. Difficulties during insertion of the AcryS of single-piece IOL. J Cataract Refract Surg. 2004;30(12):2645. Doi: 10.1016/j.jcrs.2004.09.039. PMID: 15617944.

[2] Dada T, Sethi HS, Aggarwal A. Tilt of single-piece hydrophobic intraocular lens due to folded haptic. J Cataract Refract Surgery. 2005;31(12):2429-30. Doi: 10.1016/j. jcrs.2005.08.047. PMID: 16473244.

[3] Merchant K, Stirling R. Unusual sequel to a known complication. Postgrad Med J. 2007;83(985):7. Doi: 10.1136/pgmj.2007.062455. PMID: 17989263.

[4] Hartman M, Rauser M, Brucks M, Chalam KV. Evaluation of anterior capsular contraction syndrome after cataract surgery with commonly used intraocular lenses. Clin Ophthalmol. 2018;12:1399-403. Doi: 10.2147/OPTH.S172251. PMID: 30122893.

[5] Mehta R, Aref AA. Intraocular lens implantation in the ciliary sulcus: Challenges and risks. Clin Ophthalmol. 2019;13:2317-23. Doi: 10.2147/OPTH.S205148. PMID: 31819356.

[6] Mohebbi M, Bashiri SA, Mohammadi SF, Samet B, Ghassemi F, Ashrafi E, et al. Outcome of single-piece intraocular lens sulcus implantation following posterior capsular rupture during phacoemulsification. J Ophthalmic Vis Res. 2017;12(3):275-80. Doi: 10.4103/jovr.jovr_181_15. PMID: 28791060.

[7] Chang DF, Masket S, Miller KM, Braga-Mele R, Little BC, Mamalis N, et al. Complications of sulcus placement of single-piece acrylic intraocular lenses: Recommendations for backup IOL implantation following posterior capsule rupture. J Cataract Refract Surg. 2009;35(8):1445-58. Doi: 10.1016/j. jcrs.2009.04.027. PMID: 19631134.

[8] Taskapili M, Engin G, Kaya G, Kucuksahin H, Kocabora MS, Yilmazli C. Singlepiece foldable acrylic intraocular lens implantation in the sulcus in eyes with posterior capsule tear during phacoemulsification. J Cataract Refract Surg 2005;31(8):1593-97. Doi: 10.1016/j.jcrs.2005.01.029. PMID: 16129297.

[9] Taskapili M, Gulkilik G, Kocabora MS, Ozsutcu M, Yilmazli C, Kaya G, et al. Comparison of sulcus implantation of single-piece hydrophilic foldable acrylic and polymethylmethacrylate intraocular lenses in eyes with posterior capsule tear during phacoemulsification surgery. Eur J Ophthalmol. 2007;17(4):595-600. Doi: 10.1177/112067210701700418. PMID: 17671936.

[10] Kim SM, Choi S. Clinical efficacy and complications of intraocular lens exchange for opacified intraocular lenses. Korean J Ophthalmol. 2008;22(4):228-35. Doi: 10.3341/kjo.2008.22.4.228. 19096239. 


\section{PARTICULARS OF CONTRIBUTORS:}

1. Senior Resident, Department of Ophthalmology, Sri Guru Ram Das Institute of Medical Sciences and Research, Amritsar, Punjab, India

2. Professor, Department of Ophthalmology, Sri Guru Ram Das Institute of Medical Sciences and Research, Amritsar, Punjab, India.

NAME, ADDRESS, E-MAIL ID OF THE CORRESPONDING AUTHOR:

Pawan Prasher,

Sri Guru Ram Das Institute of Medical Sciences and Research, Amritsar, Punjab, India. E-mail: pawanprasher@yahoo.com

\section{AUTHOR DECLARATION:}

- Financial or Other Competing Interests: None

- Was informed consent obtained from the subjects involved in the study? Yes

- For any images presented appropriate consent has been obtained from the subjects. Yes
PLAGIARISM CHECKING METHODS: (Jain Het al.

- Plagiarism X-checker: Apr 20, 2020

- Manual Googling: Jun 23, 2020

- iThenticate Software: Jul 28, 2020 (11\%)
ETYMOLOGY: Author Origin

Submission: Apr 20, 2020 Date of Peer Review: May 29, 2020

Date of Acceptance: Jun 23, 2020 Date of Publishing: Aug 01, 2020 